Hanxiang Chen, Department of Mathematics, University of Alabama 131037 th St. E. Apt. 92, Tuscaloosa, AL 35405,

e-mail:hchen30ua1vm . ua . edu

\title{
A PAIR OF ADJOINT CLASSES OF RIEMANN-STIELTJES INTEGRABLE FUNCTIONS
}

\begin{abstract}
The purpose of this paper is to show that the classes of Riemann integrable functions and absolutely continuous functions are adjoint with respect to the $(R-S)$ integral $\int_{a}^{b} f d g$.
\end{abstract}

Definition. Let $A$ and $B$ be two classes of functions defined on $[a, b] . A$ and $B$ are said to be adjoint with respect to the $(R-S) \int_{a}^{b} f d g$, if the following conditions are satisfied:

(i) If $f \in A$ and $g \in B$, then the $(R-S) \int_{a}^{b} f d g$ exists;

(ii) If the $(R-S) \int_{a}^{b} f d g$ exists for all $g \in B$, then $f \in A$; and

(iii) If the $(R-S) \int_{a}^{b} f d g$ exists for all $f \in A$, then $g \in B$.

If $A$ and $B$ are adjoint with respect to the $(R-S) \int_{a}^{b} f d g$, this means that on condition that the $(R-S) \int_{a}^{b} f d g$ exists, neither $A$ nor $B$ can be extended at all. For convenience, we write $(A * B) \int_{a}^{b} f d g$ meaning that $A$ and $B$ are adjoint with respect to the $(R-S) \int_{a}^{b} f d g$.

We introduce the following symbols for some classes of functions defined on $[a, b]$ :

Key Words: adjoint classes, Riemann integrable functions, the Riemann-Stieltjes integral, functions of bounded variation, absolutely continuous functions

Mathematical Reviews subject classification: 26A42

Received by the editors May 26, 1996 
$R[a, b] \quad$ class of Riemann integrable functions on $[a, b]$

$C[a, b]$ class of continuous functions on $[a, b]$

$B V[a, b]$ class of functions of bounded variation on $[a, b]$

$A C[a, b]$ class of absolutely continuous functions on $[a, b]$.

It is known [1] that $C[a, b]$ and $B V[a, b]$ are adjoint with respect to the $(R-S) \int_{a}^{b} f d g$. In this paper we would like to show that $R[a, b]$ and $A C[a, b]$ are adjoint with respect to the $(R-S) \int_{a}^{b} f d g$. To do this, we should prove that $R[a, b]$ and $A C[a, b]$ satisfy the three conditions in the definition.

(i) If $f \in R[a, b]$ and $g \in A C[a, b]$, then $(R-S) \int_{a}^{b} f d g$ exists (cf. [3] for proof);

(ii) If $(R-S) \int_{a}^{b} f d g$ exists for all $g \in A C[a, b]$, then $f \in R[a, b]$. As a matter of fact, taking $g(x)=x \in A C[a, b]$ gives that $f \in R[a, b]$.

In order to prove statement (iii) "if the $(R-S) \int_{a}^{b} f d g$ exists for all $f \in$ $R[a, b]$, then $g \in A C[a, b]$ ", we need the following lemmas.

Lemma 1 (Vitali). [2] Let $E$ be set of finite outer measure and $\mathcal{J}$ a collection of intervals that cover $E$ in the sense of Vitali. Then, given $\epsilon>0$, there is a finite disjoint collection $\left\{I_{1}, \ldots, I_{N}\right\}$ of intervals in $\mathcal{J}$ such that

$$
m^{*}\left[E \sim \cup_{n=1}^{N} I_{n}\right]<\epsilon .
$$

Lemma 2. Let $f$ be a function on $[a, b]$ such that $f^{\prime}=0$ a.e. Then, $f$ has the following property: (S) Given $\epsilon>0, \delta>0$, there is a finite collection $\left\{\left[y_{k}, x_{k}\right]\right\}$ of nonoverlapping intervals on $[a, b]$ such that

$$
\sum\left|x_{k}-y_{k}\right|<\delta
$$

and

$$
\sum\left|f\left(x_{k}\right)-f\left(y_{k}\right)\right|>|f(b)-f(a)|-\epsilon .
$$

Proof. Let $E \subset(a, b)$ be the set of measure $b-a$ in which $f^{\prime}(x)=0$, and $\epsilon$ and $\delta$ be arbitrary positive numbers. To each $x$ in $E$ there is an arbitrarily small interval $[x, x+h]$ contained in $(a, b)$ such that $|f(x+h)-f(x)|<\epsilon \cdot h /(b-a)$. By Lemma 1, we can find a finite collection $\left\{\left[x_{k}, y_{k+1}\right]\right\}$ of nonoverlapping intervals of this sort which cover all of $E$ except for a set of measure less than $\delta$. If we label the $x_{k}$ so that $x_{k}<x_{k+1}$, we have

$$
a=y_{o}<x_{o}<y_{1}<x_{1}<y_{2}<\cdots<y_{n-1}<x_{n-1}<y_{n}<x_{n}=b
$$

and

$$
\sum_{k=0}^{n}\left|x_{k}-y_{k}\right|<\delta
$$


Now

$$
\begin{aligned}
|f(b)-f(a)|=\left|\sum_{k=0}^{n}\left[f\left(x_{k}\right)-f\left(y_{k}\right)\right]+\sum_{k=0}^{n-1}\left[f\left(y_{k+1}\right)-f\left(x_{k}\right)\right]\right|< \\
\quad<\sum_{k=0}^{n}\left|f\left(x_{k}\right)-f\left(y_{k}\right)\right|+\epsilon .
\end{aligned}
$$

Thus

$$
\sum_{k=0}^{n}\left|f\left(x_{k}\right)-f\left(y_{k}\right)\right|>|f(b)-f(a)|-\epsilon .
$$

Note. If the function $f$ in Lemma 2 is continuous, then we can find a finite collection $\left\{\left[y_{k}, x_{k}\right]\right\}$ of nonoverlapping intervals in $(a, b)$ instead of $[a, b]$ such that the above two inequalities hold, too.

We are now in a position to prove the statement (iii):

Theorem. If the $(R-S) \int_{a}^{b} f d g$ exists for all $f \in R[a, b]$, then $g \in A C[a, b]$.

Proof. First of all, the fact that

$C[a, b] \subset R[a, b], B V[a, b] \subset R[a, b]$,

$(C * B V) \int_{a}^{b} f d g$ and the $(R-S) \int_{a}^{b} f d g$ exists for all $f \in R[a, b]$,

implies that $g \in C[a, b] \cap B V[a, b]$. So, it follows that $g=G+F$ with $G \in$ $A C[a, b]$ and $F \in C[a, b], F^{\prime}=0$ a.e. on $[a, b]$. To show $g \in A C[a, b]$, it suffices to show $F=$ const. on $[a, b]$. By hypothesis, the $(R-S) \int_{a}^{b} f d g$ exists for all $f \in R[a, b]$ and so does the $(R-S) \int_{a}^{b} f d F$. Suppose that $F(x) \neq$ const. on $[a, b]$. Then, there is a point $c \in(a, b]$ such that $F(c)-F(a) \neq 0$. For convenience, let $c=b$. We shall now construct a function $f \in R[a, b]$ such that the $(R-S) \int_{a}^{b} f d F$ does not exist.

Let $\epsilon$ be a number with $0<\epsilon<|F(b)-F(a)|$, and $\left\{\delta_{n}\right\}$ be a sequence satisfying $\delta_{n} \downarrow 0(n \rightarrow \infty)$. Since $F \in C[a, b]$ and $F^{\prime}=0$ a.e. on $[a, b]$, by the note of Lemma 2, the function $F$ has property (S). That is, for $\epsilon>0$ and $\delta_{1}>0$, there is a finite collection $\left\{\left[y_{k}^{(1)}, x_{k}^{(1)}\right]\right\}$ of nonoverlapping intervals in $(a, b)$ such that

$$
\sum_{k=0}^{n_{1}}\left|x_{k}^{(1)}-y_{k}^{(1)}\right|<\delta_{1}
$$


and

$$
\sum_{k=0}^{n_{1}}\left|F\left(x_{k}^{(1)}\right)-F\left(y_{k}^{(1)}\right)\right|>|F(b)-F(a)|-\frac{\epsilon}{2} .
$$

We call $\left\{\left[y_{k}^{(1)}, x_{k}^{(1)}\right]\right\}$ the first level of the collection of nonoverlapping intervals. Then, for every integer $h>1$, we can inductively find the $h$-th level of it, denoted by

$$
\mathcal{J}_{h}=\left\{\left[y_{k}^{(h)}, x_{k}^{(h)}\right]\right\}_{k=0, \ldots, n_{h}},
$$

such that

(1) $\sum_{k=0}^{n_{h}}\left|x_{k}^{(h)}-y_{k}^{(h)}\right|<\delta_{h}$;

(2) $\sum_{k=0}^{n_{h}}\left|F\left(x_{k}^{(h)}\right)-F\left(y_{k}^{(h)}\right)\right|>|F(b)-F(a)|-\epsilon \cdot\left(2^{h}-1\right) / 2^{h}$;

(3) $I_{h} \subset I_{h-1}^{\sim}$, where define $I_{h}=\cup_{k}\left[y_{k}^{(h)}, x_{k}^{(h)}\right]$ and $I_{h}^{\sim}=\cup_{k}\left(y_{k}^{(h)}, x_{k}^{(h)}\right)$.

Assume $\mathcal{J}_{h}$ is found. We wish to find $\mathcal{J}_{h+1}$. To do this, we shall make use of Lemma 2 repeatedly. Applying Lemma 2 on each interval $\left[y_{i}^{(h)}, x_{i}^{(h)}\right]$ in $\mathcal{J}_{h}\left(i=0,1, \ldots, n_{h}\right)$, we can find a finite collection $\left\{\left[y_{k(i)}^{(h+1)}, x_{k(i)}^{(h+1)}\right]\right\}$ of nonoverlapping intervals in $\left(y_{i}^{(h)}, x_{i}^{(h)}\right)$ such that

$$
\sum_{k(i)}\left|x_{k(i)}^{(h+1)}-y_{k(i)}^{(h+1)}\right|<\frac{\delta_{h+1}}{2^{i+1}}
$$

and

$$
\sum_{k(i)}\left|F\left(x_{k(i)}^{(h+1)}\right)-F\left(y_{k(i)}^{(h+1)}\right)\right|>\left|F\left(x_{i}^{(h)}\right)-F\left(y_{i}^{(h)}\right)\right|-\frac{\epsilon}{2^{h+i+2}} .
$$

Collecting all of

$$
\left\{\left[y_{k(i)}^{(h+1)}, x_{k(i)}^{(h+1)}\right]\right\} \quad\left(i=0,1, \ldots, n_{h}\right)
$$

gives us a finite collection of nonoverlapping intervals in $I_{h}^{\sim}$, denoted by

$$
\mathcal{J}_{h+1}=\left\{\left[y_{k}^{(h+1)}, x_{k}^{(h+1)}\right]\right\}_{k=0, \ldots, n_{h+1}} .
$$

Then, we have that

$$
\sum_{k=0}^{n_{h+1}}\left|x_{k}^{(h+1)}-y_{k}^{(h+1)}\right|<\delta_{h+1}\left(\frac{1}{2}+\cdots+\frac{1}{2^{n_{h}+1}}\right)<\delta_{h+1}
$$


and

$$
\begin{gathered}
\sum_{k=0}^{n_{h+1}}\left|F\left(x_{k}^{(h+1)}\right)-F\left(y_{k}^{(h+1)}\right)\right|> \\
>\sum_{k=0}^{n_{h}}\left|F\left(x_{k}^{(h)}\right)-F\left(y_{k}^{(h)}\right)\right|-\epsilon\left(\frac{1}{2^{h+2}}+\cdots+\frac{1}{2^{n_{h}+h+2}}\right) \\
>|F(b)-F(a)|-\frac{\epsilon\left(2^{h}-1\right)}{2^{h}}-\frac{\epsilon}{2^{h+1}} \\
=|F(b)-F(a)|-\frac{\epsilon\left(2^{h+1}-1\right)}{2^{h+1}} .
\end{gathered}
$$

It is clearly true that $I_{h+1} \subset I_{h}^{\sim}$.

Therefore, the above $\mathcal{J}_{h+1}$ is indeed the $(h+1)$-th level of the finite collection of nonoverlapping intervals with properties (1), (2) and (3).

We now define a function $f_{1}$ on $[a, b]$ by

$$
f_{1}(x)= \begin{cases}\operatorname{sign}\left[F\left(x_{k}^{(h)}\right)-F\left(y_{k}^{(h)}\right)\right] & \text { if } x=y_{k}^{(h)} \text { for } h \geq 1 \text { and } k=0,1, \ldots, n_{h} \\ 0 & \text { if } x \in[a, b] \sim \cup_{k, h}\left\{y_{k}^{(h)}\right\} .\end{cases}
$$

Since $I_{h+1} \subset I_{h}^{\sim}$ and $\mathcal{J}_{h}$ is the collection of nonoverlapping intervals for $h \geq 1$, and so $y_{k}^{(h)} \neq y_{j}^{(i)}$ if $(h, k) \neq(i, j)$. Whence, the function $f_{1}$ is well defined on $[a, b]$. We must show $f_{1} \in R[a, b]$. If $x_{o} \in I_{h}^{\sim} \sim I_{h+1}$ for an integer $h \geq 0$ (denote $\left.I_{0}=[a, b]\right)$, then in view of the definition of $f_{1}$, there is an open interval $O\left(x_{0}, \eta\right)=\left(x_{0}-\eta, x_{0}+\eta\right) \subset I_{h}^{\sim} \sim I_{h+1}$ such that $f_{1}(x)=0$ if $x \in O\left(x_{0}, \eta\right)$. Thus $f_{1}$ is continuous at $x_{0}$. Let $E$ be the set of this sort of points $x_{0}$. It is not hard to see that

$$
E=(a, b) \sim \cap_{h=0}^{\infty} I_{h} \sim \cup_{k, h}\left\{y_{k}^{(h)}\right\} \sim \cup_{k, h}\left\{x_{k}^{(h)}\right\},
$$

where the last two terms are countable sets.

Also, from

$$
m\left(I_{h}\right)=\sum_{k=0}^{n_{h}}\left|x_{k}^{(h)}-y_{k}^{(h)}\right|<\delta_{h} \rightarrow 0 \quad(h \rightarrow \infty)
$$

and

$$
I_{h+1} \subset I_{h} \quad(h \geq 0)
$$

we have

$$
m\left(\cap_{h=0}^{\infty} I_{h}\right)=0
$$

and so $m(E)=b-a$. 
Hence, the bounded function $f_{1}$ is continuous almost everywhere on $[a, b]$, that is, $f_{1} \in R[a, b]$. We shall now show that the $(R-S) \int_{a}^{b} f_{1} d F$ does not exist.

Given $\lambda>0$. There is a positive integer $h$ such that $0<\delta_{h}<\lambda$. Let $P$ be a subdivision, $a=x_{0}<x_{1}<\cdots<x_{n}=b$, of $[a, b]$ with $\max _{i}\left\{\Delta x_{i}\right\}<\lambda$ such that each interval $\left[y_{k}^{(h)}, x_{k}^{(h)}\right]$ of $\mathcal{J}_{h}$ is one of the subintervals of $P$. Let $\sigma$ be a Stieltjes sum, corresponding to $P$. Then, $\sigma=\sum_{i=0}^{n-1} f_{1}\left(\xi_{i}\right)\left[F\left(x_{i+1}\right)-\right.$ $\left.F\left(x_{i}\right)\right]$, where $\xi_{i} \in\left[x_{i}, x_{i+1}\right]$. If $\left[x_{i}, x_{i+1}\right]=\left[y_{k}^{(h)}, x_{k}^{(h)}\right]$, then we choose $\xi_{i}=y_{k}^{(h)}$. Otherwise, there is a point $\xi_{i} \in\left[x_{i}, x_{i+1}\right]$ such that $f_{1}\left(\xi_{i}\right)=0$. Thus, we have that

$$
\begin{aligned}
\sigma & =\sum_{k=0}^{n_{h}} \operatorname{sign}\left[F\left(x_{k}^{(h)}\right)-F\left(y_{k}^{(h)}\right)\right] \cdot\left[F\left(x_{k}^{(h)}\right)-F\left(y_{k}^{(h)}\right)\right] \\
& =\sum_{k=0}^{n_{h}}\left|F\left(x_{k}^{(h)}\right)-F\left(y_{k}^{(h)}\right)\right| \\
& >|F(b)-F(a)|-\frac{\epsilon\left(2^{h}-1\right)}{2^{h}} \\
& >|F(b)-F(a)|-\epsilon>0
\end{aligned}
$$

On the other hand, however, if we choose $\xi_{i} \in\left[x_{i}, x_{i+1}\right]$ such that $f_{1}\left(\xi_{i}\right)=0$ for $i=0,1, \ldots, n-1$, then, this leads to another Stieltjes sum $\sigma_{1}=0$. The fact that when $\lambda \rightarrow 0, \sigma-\sigma_{1} \geq|F(b)-F(a)|-\epsilon>0$ implies the $(R-S) \int_{a}^{b} f_{1} d F$ does not exist. This contradicts that the $(R-S) \int_{a}^{b} f d F$ exists for all $f \in R[a, b]$. Hence, $F=$ const. on $[a, b]$, and so $g=G+F$ is absolutely continuous on $[a, b]$. Thus the theorem is proved.

Consequently, we see that $R[a, b]$ and $A C[a, b]$ are adjoint with respect to the $(R-S) \int_{a}^{b} f d g$.

\section{References}

[1] T. H. Hildebrandt, Introduction to the Theory of Integration, Academic Press, New York and London, 1963, p. 56.

[2] H. L. Royden, Real Analysis, 3 ed., New York, London, Macmillan, 1988.

[3] K. R. Stromberg, An introduction to classical Real Analysis, 1981. 